\title{
ON THE DIRECT PRODUCT OF OPERATOR ALGEBRAS IV
}

\author{
TAKASI TURUMARU
}

(Received April 1, 1956)

M. Nakamura [2] has discussed the relation between the direct product and the generation of two sub-factors in a finite $W^{*}$-factor, and obtained a satisfactorily analogous result as the classical theory of hypercomplex numbers: that is, in a finite $W^{*}$-factor, the direct product (in $W^{*}$-sense) of elementwise commutative two sub-factors means the generation in weak operator topology and vice versa. In the present paper, we shall consider the same problem for sub-algebras in an arbitrary $\boldsymbol{C}^{*}$-algebra. In the case of $W^{*}$-factor, the key point of Nakamura's argument is the multiplicativity of the faithful normal trace. In view of this fact, we introduce a notion of independence of sub-algebras in a $C^{*}$-algebra $(\S 1$, Def.): this definition seems to be artificial at a first glance but this may be considered as a generalization of the stochastic independence in the probability theory. Finally in $\$ 2$, we shall state a theorem for an abelian $C^{*}$-algebra recently obtained by R. McDowell for real case [1].

1. Let $A$ be a $C^{*}$-algebra with the unit 1 , and $A_{i}(i=1,2)$ be two $C^{*}$-sub-algebras of $A$ which contain 1 . Then $A_{i}$ are called algebraically independent if they have following properties:

(1) $A_{1}$ and $A_{2}$ commute elementwise,

(2) If $\left\{a_{i} \mid i=1, \ldots, m\right\}$ and $\left\{b_{j} \mid j=1, \ldots, n\right\}$ are arbitrary linearly independent set of elements of $A_{1}$ and $A_{2}$ respectively, then $\left\{a_{i} b_{j} \mid i=1, \ldots\right.$, $m ; j=1, \ldots, n\}$ is linearly independent in $A$.

If $A_{i}(i=1,2)$ are algebraically independent $C^{*}$-sub-algebras of a $C^{*}$-algebra $A$, the following facts are easily verified :

(I) The algebraic direct product $A_{1} \odot A_{2}$ of $A_{1}$ and $A_{2}$ is isomorphic to the *-algebra $A_{0}$ generated by $A_{1}$ and $A_{2}$ algebraically and this isomorphism is given by the correspondence

$$
\sum_{i} a_{i} b_{i} \leftrightarrow \sum_{i} a_{i} \times b_{i} .
$$

(II) For any pair of positive linear functionals $(\sigma, \tau)$ where $\sigma$ is defined on $A_{1}$ and $\tau$ on $A_{2}$, the functional which we call a product functional of $\sigma$ and $\tau$,

$$
[\sigma \odot \tau]\left(\sum_{i} a_{i} b_{i}\right)=\sum_{i} \sigma\left(a_{i}\right) \tau\left(b_{i}\right), \sum_{i} a_{i} b_{i} \in A_{0}
$$

is well-defined on $A_{0}$ and additive, homogeneous and positive :

$$
[\sigma \odot \tau]\left(\left(\sum_{i} a_{i} b_{i}\right)\left(\sum_{i} a_{i} b_{i}\right)^{*}\right) \geqq 0, \text { for all } \sum_{i} a_{i} b_{i} \in A_{0} .
$$


Then, "Under what topological conditions for $A_{i}$, the product functional does become continuous?" is an interesting problem for us. In abelian real case, $R$. MacDowell has discussed this problem with different aspects [1]. In $\$ 2$, we shall come back to this problem for the abelian complex case. In this section we discuss the relation between this problem and the direct product $A_{1} \times$ a $A_{2}$ (cf. [4]). For this purpose, we want to introduce the notion of independence of sub-algebras. Now, we begin with the consideration on the probability theory. If $(X, F, \mu)$ be a probability measure space, then two bounded real random variables $a$ and $b$ are called mutually independent if $E(f(a)$ $g(b))=E(f(a)) E(g(b))$, where $f(\lambda), \quad g(\lambda)$ are arbitrary complex-valued Baire functions and $E(c)=\int c(x) d \mu(x)$. And moreover, the space $L^{\infty}(X, F, \mu)$ of all bounded random variables forms a commutative $W^{*}$-algebra, acting on the Hilbert space $L^{2}(X, F, \mu)$, having a faithful normal trace $E(\bullet)$. Conversely, if a commutative $W^{*}$-algebra $M$ has a faithful normal trace $\tau$, then GelfandNeumark's respresentation theorem shows that there is a probability measure space on which the algebra $M$ is isomorphically, preserving the value of the trace, represented to the algebra of all bounded random variables of the space, i. e., $\tau(a)=E\left(a^{\#}\right), a^{\#}$ denotes the representation of $a$. Since the $W^{*}$-sub-algebra $A$ generated by an hermitian element $a$ and 1 in $M$ consists of all Baire functions of $a$, the above notion of independence can be transfered into any commutative $W^{*}$-algebra $M$ with a faithful normal trace $\tau$ as follows : Two hermitian elements $a$ and $b$ are called mutually independent if $\tau(u v)=\tau(u) \tau(v)$ for every $u \in A, v \in B$, where $A$ and $B$ are $W^{*}$-sub-algebras generated by $\{a, 1\}$ and $\{b, 1\}$ respectively.

Under these considerations we set the following definition for $C^{*}$. algebras.

Definition. Let $A$ be a $C^{*}$-algebra, and $A_{i}(i=1,2)$ be algebraically independent sub-algebras. Then $A_{i}$ are called mutually independent if the product functional $\sigma \odot \tau$ is continuous on $A$ and the totality of its continuous extension $\sigma \times \tau$ is complete on the $C^{*}$-sub-algebra generated by $A_{i}(i=1,2)$.

Now, our aimed proposition is the following:

Proposition 1. Let $A$ be a $C^{*}$-algebra, $A_{i}(i=1,2)$ be $C^{*}$-sub-algebras of $A$, and if $A$ is generated by $A_{i}$, then the following two statements are mutually equivalent :

(1) $A_{i}(i=1,2)$ are mutually independent,

(2) $A$ is isomorphic to the direct product $A_{1} \times_{\alpha} A_{2}$ of $A_{i}$.

PROof. Implication (1) $\rightarrow(2)$. It is sufficient to prove that the isomorphism $\sum_{i} a_{i} b_{i} \hookleftarrow \rightarrow \sum_{i} a_{i} \times b_{i}$ between $A_{0}$ and $A_{1} \odot A_{2}$ described in (I) is isometric.

Since $A_{i}$ are mutually independent and generate $A$, the totality of $\sigma \times$ $\tau$, where $\sigma$ and $\tau$ are pure states of $A_{1}$ and $A_{2}$ respectively, is complete on $\boldsymbol{A}$, and we have by [4] 


$$
\begin{aligned}
\left\|\sum_{i} a_{i} b_{i}\right\|^{2} & =\sup _{\sigma \times \tau} \frac{[\sigma \times \tau]\left(\left(\sum_{j} a_{j}^{\prime} b_{j}^{\prime}\right)\left(\sum_{i} a_{i} b_{i}\right)\left(\sum_{i} a_{i} b_{i}\right)^{*}\left(\sum_{j} a_{j}^{\prime} b_{j}^{\prime}\right)^{*}\right)}{[\sigma \times \tau]\left(\left(\sum_{j} a_{j}^{\prime} b_{j}^{\prime}\right)\left(\Sigma_{j} a_{j}^{\prime} b_{j}^{\prime}\right)^{*}\right)} \\
& =\alpha\left(\sum_{i} a_{i} \times b_{i}\right)^{2} .
\end{aligned}
$$

Implication (2) $\rightarrow$ (1). Since $A \simeq A_{1} \times_{a} A_{2}$, then $A_{1} \simeq A_{1} \times 1, A_{2} \simeq 1 \times A_{2}$; hence $A_{1}$ and $A_{2}$ are algebraically independent, and finally the continuity of the product functional is clear from the definition of norm in $A_{1} \times{ }_{a} A_{2}$. [cf. 4]

Q.E.D.

For infinitely many sub-algebras we can prove the following

Proposition 2. Let $A$ and $A_{i}(i \in I)$ be $C^{*}$-algebras with units, then $A$ is isomorphic to the infinite product of $A_{i}(i \in I)$ in the sense of Takeda [3], if and only if there exists a mapping $\Phi$ from the set union $\bigcup_{i \in I} A_{i}$ of $A_{i}$ into $A$ such that

(1) Restriction $\phi_{i}$ of $\Phi$ on $A_{i}$ is the principal isomorphism of $A_{i}$ into $A$,

(2) Any finite set of images $\left\{\phi_{i k}\left(A_{i k}\right) \mid k=1, \ldots, n\right\}$ are mutually independent,

(3) $\left\{\phi_{i}\left(A_{i}\right) \mid i \in I\right\}$ generate $A$ as $C^{*}$-algebra.

Proof. For any finite subset of indices $\gamma=\left\{i_{1}, \ldots, i_{n}\right\}$, let $A^{\gamma}$ be the $C^{*}$-sub-algebra of $A$ generated by $\left\{\phi_{i_{k}}\left(A_{i_{k}}\right) \mid k=1, \ldots, n\right\}$, then $A^{\gamma}$ is isomorphic to $A_{i_{1}} \times \ldots \times A_{i_{n}}$ by the above Prop. 1. Then $A$ is isomorphic to the infinite direct product of $A_{i}$ by [3: Definitions 1,2].

2. In this section, we wish to answer partly for the question described in the preceding section by proving MacDowell's theorem for the complex case: that is, in an abelian case to obtain the topological condition under which product functional becomes continuous. For this purpose, we begin with the

Definition. Let $A$ be a commutative $C^{*}$-algebra with the unit, and $B$, $C$ be two $C^{*}$-sub-algebras of $A$ which contain units. Then they are called additively related sub-algebras if and only if for every pair $(b, c)$ of $b \in B$ and $c \in C$, there exists a scalar $\theta=\theta(b, c) \in I=[0,2 \pi]$ such that $\left\|b+e^{i \theta} c\right\|$ $=\|b\|+\|c\|$.

Then we have the following

THEOREM. Let $A$ be a commutative $C^{*}$-algebra with unit, and let $B, C$ be two $C^{*}$-sub-algebras of $A$. Suppose that $A$ is generated by $B$ and $C$, then the following three statements are mutually equivalent :

(1) $A$ is isomorphic to the direct product $B \times{ }_{a} C$,

(2) $B$ and $C$ are mutually independent,

(3) $B$ and $C$ are additively related.

REMARK. R. MacDowell had proved the equivalency of (1) and (3) for real case.

Proof. Since $A, B$ and $C$ are commutative $C^{*}$-algebras with the unit, 
let $C(\Omega), C(\Gamma)$ and $C(\Delta)$ be their representations on the rings of all continuous complex-valued functions on compact Hausdorff spaces respectively.

Implication (3) $\rightarrow(1)$. We use the following terminologies (MacDowell [1]): We call a subset $T$ of a Banach space $S$ a $T$-set of $S$ if $T$ is maximal with respect to the property $b_{1}, \ldots, b_{n} \in T \rightarrow\left\|\sum_{i} b_{i}\right\|=\sum_{i}\left\|b_{i}\right\|$. Moreover if $T$ is a $T$-set of $S$, then we call $\bigcup_{\theta \in T} e^{i \theta} T$ a circular $T$-set. If the space $S=C(X)$ (the space of all continuous complex valued functions on a compact Hausdorff space $X)$ then every $T$-set $T$ of $S$ has the form $\left\{f \in S \mid f\left(x_{0}\right)=e^{i \theta_{0}}\right.$ $\|f\|\}$ for some point $x_{0}$ of $X$ and scalar $\theta_{0} \in I$, and conversely every such subset is a $T$-set of $S$.

Now let $\gamma$ and $\delta$ be arbitrary pure states of $B$ and $C$ respectively, then $T_{B}(\gamma)=\{b \in B \mid b(\gamma)=\|b\|\}, \quad T_{c}(\delta)=\{c \in C \mid c(\delta)\|=\| c \|\}$ are $T$-sets of $B$ and $C$ respectively. We can prove that there exists a circular $T$-set of $A$ which contains the set $\bigcup_{\theta^{\prime} \mathrm{eI}} e^{i \theta \prime} T_{B}(\gamma) \cup \bigcup_{\theta^{\prime \prime} \in I} e^{i \theta^{\prime \prime}} T_{C}(\delta)$. Indeed, if not, for every $\theta_{0} \in I$ the set $T_{B}(\gamma) \cup e^{i \theta_{0}} T_{C}(\delta)$ can be contained in no $T$-set of $A$, hence there exist $b_{\theta_{0}} \in T_{B}(\gamma), \quad c_{\theta_{0}} \in T_{C}(\delta)$ such that $\left\|b_{\theta_{0}}+e^{s \theta_{0}} c_{\theta_{0}}\right\|<\left\|b_{\theta_{0}}\right\|+\left\|c_{0}\right\|$. Hence, there exists a neighborhood $I\left(\theta_{0}\right)$ of $\theta_{0}$ such that $\left\|b_{\theta_{0}}+e^{i \theta} c_{0} \theta\right\|\left\|b_{\theta_{0}}\right\|+\left\|c_{\theta_{0}}\right\|$ for $\theta \in I\left(\theta_{0}\right)$; and finally $I$ can be covered by a finite number of $I(\theta): I \subset \bigcup_{k=1}^{n}$ $I\left(\theta_{k}\right)$. Put $b=\sum_{i=1}^{n} b_{\theta_{i}}$ and $c=\sum_{i=1}^{n} c_{\theta_{i}}$ then $\left\|b+e^{i \theta} c\right\|<\|b\|+\|c\|$ for all $\theta$, which contradicts to the additive relatedness of $B$ and $C$.

Now, let the $\bigcup_{\theta} e^{i \theta} T_{A}(\omega)$ be a circular $T$-set of $A$ which contains the $\bigcup_{\theta^{\prime} \in \mathrm{I}} e^{\varepsilon \theta} T_{B}(\gamma) \cup \bigcup_{\theta^{\prime \prime} \in I} e^{i \theta^{\prime \prime}} T_{C}(\delta)$, then we can show $T_{B}(\gamma)=T_{A}(\omega) \cap B$ and $T_{C}(\delta)=$ $T_{A}(\omega) \cap C$. Indeed, if $b_{1}^{\prime}, b_{2}^{\prime} \in \mathrm{T}_{B}(\gamma)$ then $b_{1}^{\prime}(\omega)=e^{i \theta_{1}}\left\|b_{1}^{\prime}\right\|$ and $b_{2}^{\prime}(\omega)=e^{t \theta_{2}}\left\|b_{2}^{\prime}\right\|$ for some $\theta_{1}, \theta_{2} \in I$; hence $b_{1}=b_{1}^{\prime} /\left\|b_{1}^{\prime}\right\|$ and $b_{2}=b_{2}^{\prime} /\left\|b_{2}^{\prime}\right\|$ belong to $T_{B}(\gamma)$ and $b_{1}(\omega)$ $=e^{i \theta_{1}}$ and $b_{2}(\omega)=e^{i \theta_{2}}$. Since $b_{1}$ and $b_{2}$ belongs to $T_{B}(\gamma), b_{1}+b_{2}$ belongs to $T_{B}(\gamma)$; hence $b_{1}+b_{2} \in e^{i \theta} T_{A}(\omega)$ for some $\theta \in I$, that is $\left(b_{1}+b_{2}\right)(\omega)=e^{\theta \theta} \| b_{1}+$ $b_{2} \|=2 e^{i \theta}$. Hence $e^{i \theta_{1}}+e^{i \theta_{2}}=2 e^{i \theta}$, therefore $\theta_{1}=\theta_{2}=\theta$, and since $1 \in T_{B}(\gamma)$ belongs to $T_{A}(\omega), \theta_{1}=\theta_{2}=\theta=0$; hence $T_{B}(\gamma) \subset T_{A}(\omega)$. Similarly $T_{C}(\delta) \subset T_{A}(\omega)$. Then clearly the pure state $\omega$ of $A$ is a common extension of $\gamma$ and $\delta$, hence $\gamma \odot \delta$ can be extended continuously, that is $B$ and $C$ are mutually independent.

Implication (2) $\rightarrow(1)$. We have proved this in Prop. 1.

Implication (1) $\rightarrow(2)$. Let $b$ and $c$ be arbitrary elements of $B$ and $C$ respectively, then there exist $\theta^{\prime}, \theta^{\prime \prime} \in I, \gamma \in \mathbf{\Gamma}$ and $\delta \in \Delta$ such that $b(y)$ $=e^{t \theta^{\prime}}\|b\|, c(\delta)=e^{i \theta^{\prime \prime}}\|c\|$. Now, if we define $\theta=\theta^{\prime}-\theta^{\prime \prime}(\bmod 2 \pi), \theta \in I$ then $\|b\|+\|c\| \geqq\left\|b+e^{i \theta} c\right\| \geqq\left|\left(b+e^{\ell \theta} c\right)(\gamma, \delta)\right|=\left|b(\gamma)+e^{\theta \theta} c(\delta)\right|=\left|e^{i \theta^{\prime}}\|b\|+e^{\left(\theta+\theta^{\prime \prime}\right)}\|c\|\right|$ $=\|b\|+\|c\|$, that is, $B$ and $C$ are additively related. 


\section{BIBLIOGRAPHY}

[1] R. MACDOWELL, Banach spaces and algebras of continuous functions, Proc. Amer. Math. Soc., 6(1955), 67-78.

[2] M. NAKAMURA, On the direct product of finite factors, Tôhoku Math. Journ., 6(1954), 205-207.

[3] Z. TAKEDA, Inductive limit and infinite direct product of operator algebras, Tôhoku Math. Journ., 7(1955), 67-86.

[4] T. TURUMARU, On the direct product of operator algebras, I, Tôhoku Math. Journ., 4(1952), 242-251.

Mathematical InstituTe, TôHOKU UnIVERsity. 